\title{
L'entreprise de presse en conflit. Libération et Le Monde en restructuration
}

Conflict in press firms

\section{Camille Dupuy}

\section{(2) OpenEdition}

12 Journals

Édition électronique

URL : http://journals.openedition.org/travailemploi/4866

DOI : 10.4000/travailemploi.4866

ISSN : 1775-416X

Éditeur

DARES - Ministère du Travail

\section{Édition imprimée}

Date de publication : 15 décembre 2010

Pagination : 29-42

ISSN : 0224-4365

\section{Référence électronique}

Camille Dupuy, «L'entreprise de presse en conflit. Libération et Le Monde en restructuration », Travail et Emploi [En ligne], 124 | octobre-décembre 2010, mis en ligne le 15 décembre 2012, consulté le 20 avril 2019. URL : http://journals.openedition.org/travailemploi/4866 ; DOI : 10.4000/travailemploi.4866 


\title{
L'entreprise de presse en conflit. Libération et Le Monde en restructuration
}

\author{
Camille Dupuy (*)
}

\begin{abstract}
Cet article étudie les restructurations intervenues dans deux entreprises de presse quotidienne nationale, Libération et Le Monde, pour tenter de comprendre les dynamiques de mobilisation des salariés à un moment de conflit autour de l'emploi. Ces deux entreprises fonctionnent dans des cadres institutionnels atypiques (salariés actionnaires, clause de conscience, carte de presse) qui confèrent aux salariés un engagement double, à la fois professionnel et moral. Lors de la discussion du plan de sauvegarde de l'emploi vont se poser des questions autour de la définition du produit et des valeurs d'entreprises (actionnariat, frontières de l'entreprise, nouvelle formule rédactionnelle) au-delà des négociations classiques en termes de maintien des postes dans l'entreprise (autour du volontariat). Le droit est ainsi mobilisé par les salariés de manière à satisfaire leur éthique professionnelle (indépendance, qualité, démocratie, etc.). Et l'action des salariés semble faire passer la gestion de ce moment économique d'une rationalité en finalité à une rationalité en valeur.
\end{abstract}

La crise que traverse le secteur de la presse écrite est fréquemment analysée sous l'angle du phénomène de compression de la « double vente du journal» - aux lecteurs et aux annonceurs - (CABRolie, 2009), ou à partir de la question des « pouvoirs de la profession, son rôle politique, son devenir » (Neveu, 2004), où presse et journalistes sont considérés comme des catégories homogènes. Cet article part, quant à lui, des conditions pratiques d'exercice de l'activité journalistique et s'intéresse aux structures économiques des entreprises où s'exerce cette activité. Il adopte le point de vue d'une " sociologie économique du capitalisme moderne » (BASTIN, 2001) et se situe dans la ligne des travaux de WEBER, pour qui « le mode de fonctionnement de tout travail culturel de [la] presse aujourd'hui est lié aux conditions d'existence d'entreprises privées [...]. Il est nécessaire en d'autres termes d'examiner d'abord et avant toute autre chose l'entreprise de presse (Zeitungsgeschäft) du point de vue des conditions objectives de son existence et des répercussions de celles-ci sur le fonctionnement des types très différents les uns des autres de journaux modernes » (WEBER, 1910, reproduit dans Bastin, 2001, p. 184). La crise se manifeste par des difficultés concrètes au sein de ces entreprises, qui tentent d'y répondre de manière classique par des restructurations (plans de sauvegarde de l'emploi - ci-après PSE), comme cela a été le cas à Libération en 2006 et au Monde en 2008 ( $c f$. encadré 1).
La restructuration d'une entreprise, au-delà de sa dimension économique clairement identifiable, est un fait social, influencé par le cadre institutionnel dans lequel elle s'insère (règles juridiques, morales et tacites). Lors d'une procédure de licenciement collectif, les salariés et leurs représentants interviennent au même titre que les directions d'entreprise, par le biais du droit du travail qui leur sert à poser le cadre du débat. Les règles juridiques ne sont pas seulement alors des « règles de conduite » contraignant les comportements, mais, dans une perspective wébérienne, des «maximes pratiques » auxquelles les acteurs se réfèrent pour déterminer quelle attitude adopter parmi le champ des possibles (BERnard de Raymond, 2004). Pour renouveler une problématique souvent centrée sur « la presse et l'argent », Duval (2004) propose d'étudier l'effet des mécanismes économiques sur la production journalistique, à travers la figure du journaliste économique et de son activité au sein de l'entreprise. A contrario, nous proposons d'observer comment les salariés et leurs instances de représentation se saisissent de ces règles juridiques pour atténuer et détourner les impératifs économiques liés à la mise en place d'un plan de licenciement collectif. Ainsi, on peut envisager les cadres juridiques et institutionnels qui entourent la négociation des plans comme « des leviers pour retarder, discuter et aménager les décisions des directions d'entreprise » (DIDRY, 2007). Il s'agit alors de montrer que les institutions influencent la vie économique en ce qu'elles orientent (et non déterminent) l'action (dans des sens pluriels).

Au-delà des revendications individuelles des licenciés, une dynamique collective s'engage dans la défense de l'emploi. La diversité de ces dynamiques

(*) IDHE, ENS Cachan ; camille.dupuy@ens-cachan.fr. 
souligne l'existence d'une pluralité de «mondes de production » (SALAIS, STORPER, 1993), liés aux singularités du travail, du produit. Dans le secteur de la presse, les salariés sont fortement attachés à des valeurs professionnelles (comme c'est le cas également dans l'économie sociale). Selon Anne-France de SAINT LAURENT-Kogan (2000), « le monde de la presse est unique en son genre. De plus, chaque journal est un cas particulier $\gg$ (p. 56). Les journalistes de la " galaxie » de la presse quotidienne nationale s'engagent dans leur activité, et s'attachent à des valeurs spécifiques liées à la liberté de la presse (NEVEU, 2004). Ces salariés ont un rôle actif dans la clarification de leurs relations de travail et de leurs conditions de travail. À Libération et au Monde, ils bénéficient d'une double Capability for Voice (1) puisqu'ils sont à la fois salariés (avec des instances de représentation des personnels classiques) et actionnaires. Notre hypothèse est que l'activité de ces salariés s'appuie sur un ensemble de cadres institutionnels : carte de presse, sociétés de journalistes, droit du travail spécifique... Ces cadres leur offrent différents outils pour s'engager dans la négociation : au-delà de la recherche d'une indemnisation du licenciement, ils utilisent ces leviers pour influencer le devenir même du produit qui est le leur.

À partir des plans de licenciement collectif de ces deux entreprises, nous étudions ce que BASZANGER et DODIER désignent comme « une forme d'activité [la restructuration], explorée en plusieurs lieux » à partir de « la question du faire » (1997, p. 53). Nos résultats sont comparés à d'autres cas de restructurations répertoriés par Claude DIDRY. Cet auteur définit quatre registres d'action pour les salariés : l'«approfondissement des politiques de l'emploi » (contenu du plan), la « critique des argumentations économiques de la direction », la « résistance à la stratégie de compétitivité » (1998) et le « registre transactionnel » (relation qui se noue entre la négociation du plan de sauvegarde de l'emploi et le débat sur l'avenir économique de l'entreprise) (2007). Ces quatre registres sont autant d'idéaux-types qui ne reflètent pas « la réalité empirique, mais un construit mental » à partir duquel les écarts entre cas concret et modèle peuvent se mesurer (COENEN-HUTHER, 2006).

En quoi la mobilisation du droit par les instances de représentation au sein de ces dynamiques d'action collective, révèle-t-elle des particularités reliées à une éthique professionnelle, en comparaison avec des processus similaires dans d'autres entreprises ?

Nous partirons du contexte institutionnel dans lequel ces restructurations ont été menées, avant de nous intéresser à la restructuration comme épreuve et remise en cause de la communauté des salariés. Enfin, nous étudierons la manière dont les salariés

(1) D'après le concept de capability développé chez Amartya SEN in L'idée de justice, Paris 2010, Flammarion. sont porteurs d'innovation dans les moments de crise. Cela nous permettra de comprendre les dynamiques de l'action collective dans ces deux entreprises.

\section{Encadré 1 \\ L'enquête auprès de Libération et du Monde}

Cette recherche se fonde sur deux monographies d'entreprise, étudiées à deux moments différents du processus de restructuration : Libération a été étudié au moment de la négociation du plan social, alors que $\boldsymbol{L} \boldsymbol{e}$ Monde a été étudié après les négociations (au moment de l'application du plan social).

Le travail de terrain a consisté en la conduite de dix-neuf entretiens semi-directifs au cours desquels il a été demandé aux enquêtés de raconter le processus de restructuration. Bien que la population salariée de ces deux entreprises ne soit pas homogène, la population des enquêtés est constituée des acteurs qui prennent part à l'élaboration, à la discussion, et à la mise en place des plans de sauvegarde de l'emploi, et qui de ce fait, produisent un discours à même d'intégrer leur diversité. Ainsi, on suppose que l'action des instances de représentation du personnel représente la volonté du groupe dans son ensemble. Les enquêtés sont :

- Des journalistes :

- les délégués syndicaux des syndicats représentatifs : SNJ, CFDT, SNJ-CGT (et SUD à Libération) ;

- les secrétaires du comité d'entreprise (CE) ;

- les délégués du personnel, notamment les présidents (et anciens présidents) des sociétés de journalistes (association regroupant les salariés) : Société civile des personnels de Libération (SCPL), Société des rédacteurs du Monde (SRM) ;

- des journalistes qui ont choisi de partir au moment du plan social.

Signalons que les journalistes ne sont pas les seuls salariés dans les entreprises de presse. Les cadres, ouvriers et employés sont eux aussi représentés et concernés par les plans sociaux.

- Des non-journalistes :

- les présidents de la Société des cadres (du Monde) et de la Société des employés (du Monde);

- les conseillers du comité d'entreprise : experts et avocats ;

- des membres de la direction : ressources humaines et préparateurs des plans.

Au vu des responsabilités qu'endossent les enquêtés, ils ont tous plus de quarante ans et de l'ancienneté dans l'entreprise. La répartition en termes de genre est égale.

Ces récits ont été croisés avec une revue de la presse, ces deux conflits ayant été largement commentés dans les médias, ce qui nous a permis de construire une chronologie précise des événements. Nous avons également utilisé des documents mis à notre disposition par les comités d'entreprise et leurs experts (bilans annuels financiers et sociaux). 


\section{Des restructurations dans un cadre singulier : journalistes et entreprises de presse}

Les changements au sein de Libération et du Monde ne peuvent se réduire à une décision unilatérale des directions. Le débat s'est appuyé sur des formes spécifiques de représentation des salariés, dont les mobilisations collectives s'inscrivent dans un cadre institutionnel singulier : celui des entreprises de presse. Le licenciement économique, en tant que moment de crise, permet d'en faire ressortir les traits saillants.

\section{Travailler dans une entreprise de presse}

La profession de journaliste s'est constituée en référence à une éthique professionnelle, celle de la liberté d'expression et de la place des médias dans le processus démocratique. «Le journaliste n'est pas un salarié intellectuel ordinaire, pas plus que le journal n'est une denrée assimilable aux autres » explique Émile Brachard, parlementaire à l'origine de la loi sur le journaliste professionnel (DELPORTE, 1999). Le métier de journaliste est régi par l'article 7111-3 du Code du travail (2). Son statut est délimité par la carte d'identité professionnelle des journalistes (dite « carte de presse »), qui restreint l'accès à la profession. La communauté journalistique se regroupe autour de cette carte, perçue comme « la marque de l'appartenance à une communauté, une confraternité, avec ses règles et sa déontologie [chartes de 1918, de 1971...] » (DA LAGE (3), 2003).

La particularité juridique de cette profession réside dans ce que l'on appelle la «clause de conscience », signe du caractère moral (et non seulement financier) qui lie le salarié à son entreprise. Un journaliste qui rompt le contrat de travail en s'appuyant sur les motifs visés à l'article L. 7112-5 du Code du travail : « Cession du journal, cessation de la publication du journal, ou changement notable dans le caractère ou l'orientation du journal » a droit aux indemnités prévues lors d'un licenciement. Le contrat de travail engage donc plus que l'échange d'un service (rédaction d'articles) en contrepartie d'un salaire. C'est « l'honneur, la réputation ou, d'une manière générale, les intérêts moraux (4) » qui sont en jeu dans le contrat de travail, puisqu'il peut être rompu pour ces motifs. L'investissement journalistique est double : à la fois professionnel et moral.

La revendication d'indépendance est formulée de manière récurrente dans le monde de la presse, notamment dans la presse quotidienne nationale, qui se réclame d'un « magistère moral » (CHAMPAGNE,

(2) Loi Brachard de 1935, article L. 7111-3 du Code du travail. (3) Ancien président de la commission de la carte d'identité professionnelle des journalistes (commission paritaire en charge de la délivrance de la carte de presse).

(4) Article L. 7112-5 du Code du travail.
2000). Ces principes, défendus comme des principes absolus par les salariés, sont en fait des principes contingents, « historiquement construits et quotidiennement retravaillés » selon l'étude de LEMIEUX (2000). Les journalistes constituent toutefois bien le cœur d'une entreprise de presse, même en présence d'une conception actionnariale de l'entreprise : « La valeur de la marque qui les intéresse, eux, grands financiers ou patrons, elle est intrinsèquement liée à la qualité du travail du journaliste ». (Entretien avec une journaliste de Libération).

Mais les cadres, ouvriers et employés sont eux aussi représentés et concernés par les plans sociaux : par exemple, sur les 129 départs au Monde, 89 concernent ces catégories de personnels (cf. infra). Au Monde, ce sont les non-journalistes qui déterminent la couleur syndicale du secrétariat du comité d'entreprise (la CGT est minoritaire chez les journalistes, mais majoritaire dans les deux autres collèges). Ils semblent partager les mêmes valeurs que les journalistes : «Par nature, les journalistes y sont plus attachés bien sûr, mais on n'est quand même pas une entreprise comme les autres. Aussi bien chez les cadres que chez les employés, il y a la conscience que le journal est un outil démocratique. » (Entretien avec un employé du Monde.)

Bien que les salariés du Monde et de Libération soient hétérogènes ( $c f$. infra), leurs instances de représentation les engagent collectivement dans la défense de ces valeurs.

\section{Deux entreprises hors du commun}

Le Monde est créé en 1944 par Hubert BeuveMéry qui en fait une « aventure (5) » intellectuelle, morale et rédactionnelle. Ce journal s'affirme comme un journal de référence, exhaustif et indépendant, un journal « au service de l'intérêt public » (Eveno, 2004). Trois décennies plus tard, et dans un contexte différent, Libération naît de la volonté de militants maoïstes de « donner la parole au peuple » (Guisnel, 2003). Ces deux entreprises vont lutter pour la garantie d'une indépendance financière, gage de l'indépendance éditoriale (6). Les histoires du Monde et de Libération sont marquées par une forme d' « anticapitalisme » (Duval, 2004) qui va être remis en cause à partir du début des années 1980 , avec la multiplication de difficultés économiques qui conduiront à l'ouverture de leur capital. L'arrivée d'actionnaires extérieurs privés dans ces entreprises autofinancées pose des problèmes éthiques. Chacune des entreprises va adopter une forme particulière

(5) Hubert Beuve-Méry emploie de préférence pour qualifier le journal le terme d' «aventure » plutôt que celui d' « entreprise » d'après EvENo, 1997.

(6) L'idée partagée par les salariés est que le pouvoir de décision doit appartenir aux salariés (et non aux actionnaires extérieurs) pour éviter tout risque de collusion (avec les pouvoirs économiques et politiques notamment). 
Encadré 2

\section{Structure simplifiée du Groupe La Vie-Le Monde}

Les recapitalisations se font au niveau du Monde SA. Malgré la dilution du capital, les actionnaires au niveau de Le Monde et Partenaires associés (LMPA) (actionnaires internes et externes) voient donc leur pouvoir politique se maintenir. Ainsi, les actionnaires internes de LMPA (et notamment la Société des rédacteurs du Monde) restent majoritaires dans le capital de la Société éditrice du Monde (SEM) (par l'intermédiaire du Monde SA) malgré l'arrivée de nouveaux investisseurs. La SEM est la structure porteuse des filiales et bénéficie directement des revenus qui en sont tirés.

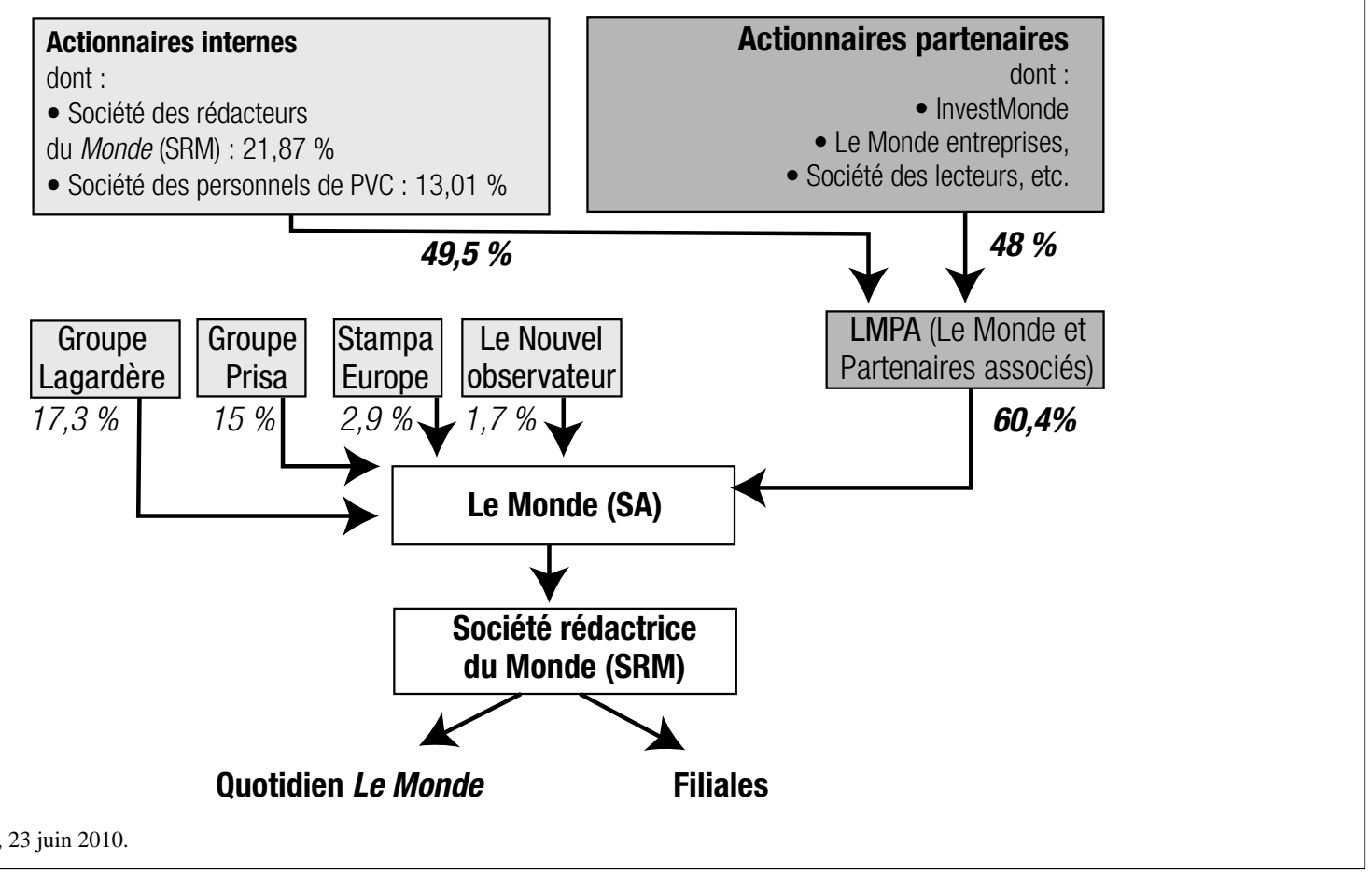

pour tenter d'y répondre. Les structures juridiques complexes qui en résultent sont la manifestation de la volonté d'indépendance des journalistes face aux actionnaires. «Moi, j'ai une vision encore impériale, c'est-à-dire qu'il ne faut pas perdre de vue que, tout ça [l'architecture du Groupe], a été fait pour l'indépendance du journal » raconte un journaliste du Monde.

Une Unité économique et sociale (7) (UES) est crée à Libération en 1981. Cette structure permet d'intégrer le journal Libération, Espace Libération, la régie publicitaire, et Paris Action Presse, la société de portage. Ces trois sociétés répondent aux différents critères établis par le Code du travail pour la reconnaissance d'une UES : une unité économique (caractérisée par la complémentarité des activités et la concentration des pouvoirs de direction) et une unité sociale (caractérisée par une gestion commune des personnels et des conditions d'exécution du travail similaires) (8). La mise en œuvre d'une UES

(7) C'est en général un arrêt de la chambre criminelle du 23 avril 1970, décidant que des sociétés peuvent avoir une existence juridique propre, et ne pas constituer à l'égard du droit du travail des entreprises distinctes, qui est considéré comme l'arrêt fondateur de l'UES.

(8) Le juge doit relever tout à la fois l'existence d'une unité sociale et d'une unité économique (Soc. 29 avril 1981, $\left.\mathrm{n}^{\circ} 80-60.374\right)$. implique la mise en place d'instances représentatives du personnel plus nombreuses (9), offrant une meilleure garantie de l'indépendance des salariés.

Dans le cas du Monde, un Groupe de presse à l'architecture complexe (La Vie-Le Monde) est créé sous la tutelle d'Alain Minc en 2001 : le quotidien y apparaît comme le rouage central ( $c f$. encadré 2 ). Les salariés du quotidien conservent la primauté décisionnelle sur l'ensemble du Groupe, grâce aux apports financiers des actionnaires extérieurs. Le Groupe réalise des acquisitions dans le domaine de la presse régionale et magazine, en se cantonnant à la recherche de valeurs financièrement rentables (« vaches à lait » selon certains salariés du quotidien). Il n'y a pas de perspective d'intégration de ces autres titres dans une ligne éditoriale commune. Le Groupe est un moyen pour le journal d'assurer son indépendance et de supporter les déficits récurrents du quotidien, par la mise à disposition d'actifs financiers, et au prix d'un comportement de « prédateur», parfois, à l'égard des autres titres du Groupe.

(9) Création d'un comité d'entreprise par société (pour celles regroupant au moins 50 salariés, article L. 2322-4 du Code du travail) ainsi qu'un comité d'entreprise commun à l'UES. 
Des relations salariés-dirigeants qui se normalisent

Les plans de licenciement collectif se succèdent depuis les années 1980 dans ces deux entreprises. Lorsque s'ouvre, à Libération, le plan de sauvegarde de l'emploi que nous étudions (septembre 2006), la dernière réduction d'effectifs date seulement de quelques mois (mars 2006, avec la suppression de cinquante-six postes). Ce constat pose la question d'une éventuelle constitution paradoxale de « routines » dans la gestion de ces moments de crise. Cependant, un bouleversement s'est opéré entre les plans étudiés et les précédents : celui de l'identité des dirigeants. L'éviction des figures emblématiques qu'ont été Serge July (à Libération de 1973 à 2006) et dans une moindre mesure (car il ne fait pas partie des fondateurs du quotidien) Jean-Marie Colombani (au Monde de 1994 à 2007), influence la manière dont vont se dérouler les négociations. Ces deux hommes incarnaient l'entreprise et rassuraient tant les salariés que les actionnaires grâce à leur très bonne maîtrise des histoires et des habitudes de leur entreprise. Les salariés avaient confiance dans les choix des dirigeants : " Serge July a même réussi à faire voter à son équipe sa propre démission en 1981 ! ». (Entretien avec un délégué syndical de Libération).

Les liens entre les salariés et la direction éclatent sous l'effet de pressions financières qui éloignent ces dirigeants historiques. Le pouvoir est cédé à de nouvelles directions (Laurent Joffrin à Libération, Éric Fottorino et David Guiraud au Monde) qui ne disposent pas de la confiance permettant la légitimation du plan social qu'ils mènent ( $c f$. encadrés 3 et 3 bis). « On négocie un plan social avec des gens qui ne connaissent pas le quotidien, ni ses procédures, ni ses salariés » raconte un journaliste de Libération.

\section{Des salariés actionnaires}

Enfin, la particularité de ces restructurations repose sur la présence d'une instance de représentation du personnel singulière, les sociétés de participation, par lesquelles les salariés détiennent des parts du capital de l'entreprise. Sur le modèle des sociétés coopératives et participatives (SCOP), les salariés (ou sociétaires) participent à la gouvernance et à la défense des intérêts économiques de leur entreprise. Comme dans le célèbre exemple de la coopérative multinationale Fagor Electrodomésticos (Espagne), le cadre des négociations collectives classiques (syndicats/direction) est modifié, puisque les travailleurs sont eux-mêmes propriétaires et gestionnaires (pour partie) de leur entreprise (AMADO-BORTHAYRE, 2009, BOCQUET et al., 2010). Ce mode d'organisation se rencontre dans des secteurs, comme celui de la presse mais aussi de l'économie sociale, où les éthiques professionnelles sont fortement construites.

\section{Encadré 3}

\section{Chronologie et contenu du plan de sauvegarde de l'emploi (Libération)}

Avril 2005 : arrivée d'Édouard de Rothschild comme actionnaire majoritaire du quotidien.

Juin 2006 : éviction de Serge July. Ouverture d'une période de cogérance entre la SCPL (représentée par Vittorio de Filippis) et Philippe Clerget, (nommé par Édouard de Rothschild), jusqu'à la nomination de Laurent Joffrin comme PDG mi-novembre.

Septembre 2006 : après un travail d'audit des comptes, Édouard de Rothschild découvre que les pertes d'exploitation estimées pour 2006 s'élèvent à 13 millions. Il annonce alors par voie de presse son intention de présenter son propre « plan de la dernière chance $(1)$ ».

Octobre-novembre 2006 : Libération est placé en procédure de sauvegarde le 4 octobre. Le lendemain, le comité d'entreprise déclenche son droit d'alerte. La Société civile des personnels de Libération (SCPL) présente un plan de sauvegarde de l'emploi, rejeté en conseil d'administration le 26 octobre. Début novembre, Laurent Joffrin présente son plan social, accepté le 7 novembre par le conseil d'administration et quelques jours plus tard par l'assemblée générale des salariés. Il prévoit la suppression de 81 postes et la création de 5 autres, soit la suppression de 76 postes équivalent temps plein (40 postes à la rédaction écrivante, 23 à la rédaction non écrivante et 13 dans l'administratif) sur 276. L'introduction d'une nouvelle technologie (nouveau système informatique d'édition) avec le projet « Méthode » est la clef de voûte du projet et justifie la suppression de 18 postes (les plus techniques).

Décembre 2006 : procédure d'information-consultation du comité d'entreprise.

Janvier 2007 : ouverture du guichet départ. En parallèle, les statuts de l'entreprise sont modifiés. Libération est transformé en société anonyme, doté d'un conseil de surveillance et d'un directoire, ce qui conduit les personnels à abandonner le droit de veto sur les grandes décisions touchant au journal.

Mars 2007 : première vague de départs (63).

Avril 2007 : rapport Nouvelle technologie du CHSCT sur le projet « Méthode ».

Juin 2007 : seconde vague de départs (18). Sources : revue de presse, entretiens, rapports d'experts.

(1) Libération du 15 septembre 2006.

"Il y a un côté schizophrène car les salariés sont à la fois représentés en tant que salariés (CE et syndicats) et en tant qu'actionnaires (SCPL au CA) ». (Entretien avec un journaliste de Libération).

Ces structures donnent une plus large place aux salariés dans la gestion de leur entreprise. Elles sont la réponse à la volonté d'indépendance des salariés par rapport aux impératifs de rentabilité de 


\section{Encadré 3 bis \\ Chronologie et contenu du plan de sauvegarde de l'emploi (Le Monde)}

Juin 2007 : éviction de Jean-Marie Colombani, remplacé par le trinôme Philippe Jeantet (président du directoire du Groupe), Bruno Patino (directeur général du Groupe), Éric Fottorino (directeur de la SEM).

Bilan financier : la SEM est déficitaire, et ce depuis plusieurs années. Ses pertes cumulées depuis 1999 s'élèvent à 167 millions d'euros. La situation du Groupe ne permet pas de soutenir ces mauvais résultats. En effet, les résultats du Groupe sont en constante dégradation depuis 2001. Le résultat net de l'ensemble consolidé (1) est déficitaire de 15,4 millions d'euros pour 2007.

Janvier 2008 : après la démission du trinôme, Éric Fottorino est nommé président du Groupe et David Guiraud directeur général.

Avril 2008 : la direction annonce un plan de départs contraints qui se décline selon trois modalités :

- suppression de 129 postes à la SEM (89 à la rédaction dont 60 journalistes, et 40 à l'administration) sur 564, afin d'économiser 9,4 millions d'euros en année pleine sur la masse salariale. Il est envisagé de réorganiser la rédaction et de rationaliser les fonctions support en optimisant leur fonctionnement, tout en conservant une qualité rédactionnelle incontestable avec la mise en place d'une nouvelle formule ; - cession de plusieurs « entités déficitaires ou non stratégiques » vers un recentrement autour d'activités complémentaires à celles du quotidien. Les sociétés vendues fin 2008 sont Fleurus Presse, les Éditions de l'Étoile (Les Cahiers du Cinéma), SPER (Danser), La Procure. Cela s'inscrit dans une stratégie plus globale de désengagement progressif du Monde des magazines et du secteur « Livre des PVC »;

- le dernier élément concerne l'immobilier. Le site est jugé trop grand pour la société (16 $800 \mathrm{~m}^{2}$ contre un besoin estimé à $12000 \mathrm{~m}^{2}$ ). Le Monde Interactif et une partie de VM Magazine emménagent boulevard Blanqui à la rentrée 2009, afin d'économiser 2 millions d'euros en année pleine.

Trois jours de grève (14 avril, 16 avril 5 mai 2008).

Ouverture des consultations du comité d'entreprise.

Mai 2008 : la direction accepte de transformer le PSE en un plan de départs volontaires. Ouverture du « guichet départ » jusqu'au 30 juin.

Juillet 2008-mars 2009 : départs.

Sources : revue de presse, entretiens, rapports d'experts.

(1) Le chiffre d'affaires « consolidé » désigne le chiffre d'affaire de l'ensemble des sociétés d'un groupe. Il est constitué de la somme des chiffres d'affaires des filiales du groupe, après élimination des ventes internes entre filiales. l'entreprise. Dans les deux cas étudiés ici, les sociétés de participation ont été créées à des moments où la qualité ou l'indépendance du titre était menacée.

À Libération, l'ensemble du personnel est réuni dans la Société civile des personnels de Libération (SCPL), qui détient $18,45 \%$ des parts (jusqu'à la recapitalisation de décembre 2006 où sa part est réduite à $1 \%$ symbolique). Elle traduit le passage d'une démocratie directe à une démocratie représentative au terme de la crise de 1981, avec l'introduction de la publicité et la discussion autour de l'arrivée de capitaux extérieurs. Les salariés créent cette société dans le but de limiter les ingérences de l'actionnaire extérieur. La SCPL participe à un dispositif de cogérance au moment du départ de Serge July, c'est-à-dire qu' elle est pendant un temps « la direction » (de juin à novembre 2006). Elle dispose d'un droit de veto sur les décisions prises en conseil d'administration (jusqu'en décembre 2006) et influence ainsi les négociations portant sur le plan de sauvegarde de l'emploi.

De même il existe au Monde trois sociétés de personnels (rédacteurs, cadres et employés) qui disposent de quatre sièges sur vingt au conseil de surveillance (instance supérieure qui gère le Groupe La Vie-Le Monde). La société des rédacteurs du Monde (SRM) est l'actionnaire majoritaire du Groupe. Elle a été créée en 1951 pour « sauvegarder l'indépendance et la continuité du journal [en] consacr[ant] son droit à la copropriété du journal » (extrait de la pétition adressée aux actionnaires au moment de la création, EvENo, 2004). La SRM dispose d'un droit de veto sur la nomination $\mathrm{du}$ directeur du Groupe. Dans le prolongement de cette société, les sociétés des cadres (1964) et des employés (1965) se sont créées dans le but de participer, avec les journalistes, à la gestion de l'entreprise (cf. supra, encadré 2).

Dans les deux cas, ce ne sont pas seulement les journalistes, mais bien l'ensemble des personnels qui sont représentés en tant qu'actionnaires. Ces plans de sauvegarde de l'emploi sont les derniers au cours desquels les sociétés de personnels actionnaires disposent des prérogatives d'actionnaire. En effet, leur pouvoir est en délitement. La SCPL abandonne ses droits en décembre 2006, condition requise par Édouard de Rothschild pour renflouer le titre. Quand aux sociétés des personnels du Monde, elles viennent de perdre leur place d'actionnaire majoritaire, avec le rachat du Groupe par Xavier Niel, Mathieu Pigasse et Pierre Bergé en novembre 2010.

Tel est donc le cadre institutionnel particulier où vont se dérouler, pour ces deux entreprises de presse quotidienne nationale, des phases de négociation collective. En quoi ce cadre institutionnel va-t-il influencer le processus de restructuration? 


\section{Restructuration et mobilisation collective de la communauté de salariés}

Les restructurations qui se déroulent au Monde et à Libération mettent à l'épreuve la collectivité des salariés, et créent une dynamique de mobilisation autour des instances de représentation du personnel. L'étude des deux plans sociaux permet de dégager les singularités de la gestion des restructurations dans ces entreprises.

\section{La légitimation du plan par les salariés actionnaires}

Les sociétés de personnels-actionnaires jouent un rôle important en amont de la négociation du contenu du plan de sauvegarde de l'emploi. Elles interviennent au moment même de l'élaboration et de la définition des termes du plan en conseil d'administration. Dans les deux cas, les justifications économiques et marchandes avancées par la direction ne sont pas remises en cause. Salariés et dirigeants partagent l'idée que l'entreprise doit s'adapter à son environnement, et qu'elle ne peut rien pour le modifier. La solution mécanique semble être la réduction des effectifs. Le projet de licenciement n'est pas « déconstruit » (BOYER, 2005). La décision de licenciement économique paraît dictée par la nécessité alors qu'elle est le résultat d'un choix des dirigeants. Tout en acceptant (peut-être à tort) la nécessité économique de restructurer, les salariés actionnaires vont faire des contre-propositions sur les modalités d'application du plan.

«On est dans une situation économique déficitaire, donc on est d'accord sur le fait qu'aujourd' hui Libération perd tellement d'argent [...] qu'on ne peut pas faire l'économie de supprimer des emplois. Mais on en conteste l'ampleur, la façon dont il est conçu, et la philosophie dans laquelle il s'inscrit». (Entretien avec un journaliste de Libération).

À Libération, la SCPL (qui représente l'ensemble des salariés) cogère l'entreprise (depuis juin 2006) lorsque l'actionnaire principal, Édouard de Rothschild, annonce sa volonté de conduire un plan social (septembre 2006). La SCPL présente un plan alternatif en octobre, sur les propositions d'Edwy Plenel : réduction d'effectifs (66 départs) et baisse des salaires. Il est rejeté au conseil d'administration du 18 novembre 2006. Les salariés actionnaires sont donc eux-mêmes à l'initiative d'un plan de licenciements collectifs. Laurent Joffrin, nommé président du directoire le 20 novembre, présente un plan de sauvegarde de l'emploi qui suit les recommandations de l'actionnaire majoritaire. Il est accepté par l'assemblée générale de la SCPL à la fin du mois, avant que ne s'ouvre l'information-consultation légale du comité d'entreprise (le $1^{\text {er }}$ décembre).
De même, le plan de sauvegarde de l'emploi est présenté au conseil de surveillance du Monde (début avril 2008) en amont de l'ouverture des négociations avec les instances classiques de représentation du personnel. Le plan prévoit que les départs soient contraints. Or les salariés du Monde ont l'habitude des plans de départs volontaires. Ils considèrent que la rupture du contrat de travail doit être le résultat du choix du salarié. Ainsi, ils remettent en cause le lien de subordination institué par le contrat de travail. Pour ce motif, la société des employés vote contre ce plan en conseil de surveillance. Cependant, un plan de départs contraints coûte moins cher à l'entreprise qu'un plan de départs volontaires (plus compliqué à organiser et plus coûteux avec des « effets d'aubaine » pour les plus âgés). Les deux autres sociétés de personnels actionnaires sont donc prises entre la rationalité économique de l'actionnaire et l'éthique du volontariat propre aux entreprises de presse. «Culturellement, c'était très difficile de faire autre chose qu'un plan de départs volontaires, rationnellement, il fallait un plan de départs contraints » résume un membre de la SRM (au cours d'un entretien). Elles vont alors jouer sur l'ambiguïté entre « approuver » et « voter » un plan. Un dysfonctionnement dans l'ordre du jour du conseil de surveillance empêche les rédacteurs et les cadres de voter (les responsables n'ont pas été mandatés). Cela leur permet de ne pas s'exprimer sur le plan et ainsi de ne pas se désolidariser des syndicats qui appellent parallèlement à la grève contre les départs contraints. Ils réussissent à contourner la difficulté de leur position («double casquette » de salarié actionnaire) en jouant de la règle de droit. Cela leur permet de valider la tenue d'un plan d'économies sans en valider les détails.

Le fait que les salariés actionnaires acceptent la tenue d'un plan de départs constitue un préambule à l'acceptation par les autres instances de représentation du personnel de la nécessité économique d'une restructuration. C'est sur les conséquences et les modalités pratiques de ces restructurations que les salariés vont s'opposer à la direction dans le cadre de la négociation collective, mais pas sur son principe même.

\section{La mobilisation des ressources légales par le comité d'entreprise}

Lors d'un plan de sauvegarde de l'emploi, le comité d'entreprise se trouve au cœur du débat à travers la procédure d'information-consultation. Le comité d'entreprise de Libération est composé de sept membres et le secrétariat revient à la liste commune CFDT/SNJ, tandis que celui du Monde est composé de dix membres, à dominante CGT. Les élus qui ont participé à la discussion des plans précédents voient leurs fonctions évoluer après l'arrivée des nouvelles directions. Comme l'expliquent Jean-Pierre Aubert et Rachel BeAujolin-Bellet (2004), « le jeu de la consultation, pour les salariés et 
leurs représentants, vise à gagner du temps et à faire monter les enchères, éventuellement sous la pression de conflits sociaux et juridiques ». " Le but du jeu, c'est d'utiliser tout l'arsenal juridique du Code du travail » raconte un représentant de Libération. Pour cela, le comité d'entreprise dispose de deux ressources.

La première est la possibilité de nommer un expert économique (10). Dans les deux cas, les comités d'entreprise ont mandaté un cabinet d'expertise qui dispose de trois semaines pour accomplir sa mission. Le recours à un expert s'explique pour trois raisons. La première est la validation des chiffres présentés par la direction et de l'impératif économique de restructurer. Cela crée un terrain d'entente avec la direction.

"Si l'expert avait dit que tous les chiffres de la direction étaient bidons ça aurait chauffé !». (Entretien avec un membre de la direction du Monde).

Même si les salariés sont d'accord sur la nécessité d'un plan de restructuration, cela n'élimine pas toutes formes de débats. Les enjeux sont déplacés sur le côté « social» (« Livre III (11)»). La deuxième raison est un corollaire de la première : l'expert permet de construire une rhétorique et un argumentaire à même d'influencer le contenu social du plan de sauvegarde de l'emploi. Il ne permet donc pas seulement au comité d'entreprise de mieux comprendre le plan, mais aussi de donner des arguments de poids dans la discussion des mesures à prévoir au sein du plan.

"Sur le volet social, l'expert nous a donné des bons trucs pour contrer, discuter, réclamer à la direction des choses supplémentaires ». (Entretien avec un membre du comité d'entreprise du Monde).

Enfin, au-delà de l'aide que peut apporter l'expert en termes de compréhension, il permet de gagner du temps. Il y a dans ces deux entreprises la création d'une urgence par les directions qui souhaitent que les plans soient mis en place le plus rapidement possible. Or les salariés souhaitent baisser les cadences imposées par la direction. Le recours à l'expert donne un délai supplémentaire pour discuter des conditions de mise en œuvre du projet. À Libération par exemple, la procédure d'information-consultation du comité d'entreprise s'ouvre le $1^{\text {er }}$ décembre 2006. Les négociations sont gelées

(10) Financé par la direction, article L. 1233-34 du Code du travail.

(11) Cela correspond à la dénomination en vigueur au moment de notre étude, qui a disparu avec la refonte du Code du travail de mai 2008. Le Livre III définit la liste des postes supprimés, les causes économiques de ces suppressions ainsi qu'un plan de reclassement. Le Livre IV expose l'argumentaire économique et organisationnel. Il montre pourquoi la société a besoin de se restructurer et pourquoi certains postes spécifiques doivent être supprimés. en attendant que l'expert rende son rapport, le 22 décembre (12). Cela donne un délai supplémentaire aux instances de représentation du personnel pour négocier de manière informelle en parallèle avec la direction. Grâce à l'intervention de l'expert, c'est la philosophie même du plan qui va être inversée. La question première n'est plus combien il faut économiser, mais combien il faut de personnes pour faire un journal de qualité.

"Les dirigeants sont partis d'un dimensionnement d'effectifs en fonction du budget de salaire qu'on peut payer, plutôt que, pour faire un journal, il nous faut tel et tel poste, donc un raisonnement un peu à l'envers ». (Entretien avec l'expert du comité d'entreprise du Monde).

La deuxième ressource dont dispose le comité d'entreprise est celle de l'action en justice. Ce droit d'action en justice est agité comme une menace à l'égard de la direction (mais pas utilisé) pour orienter le devenir du plan de sauvegarde de l'emploi, comme l'explique l'avocat du comité d'entreprise de Libération: «La pression qu'on a pu mettre a été de nature à aider ».

Grâce à ces ressources légales, le comité d'entreprise permet de ménager un terrain d'entente avec la direction.

\section{Une négociation parallèle avec les syndicats}

Les syndicats sont l'autre acteur central dans les négociations collectives. Dans ces entreprises, les syndicats représentatifs sont le SNJ, la CGT et la CFDT, et SUD à Libération. L'union syndicale qui se manifeste par la création d'intersyndicales est décisive dans l'élaboration de revendications autour du plan de sauvegarde de l'emploi (13).

L'outil le plus remarquable et le plus efficace dont disposent les syndicats est le droit de grève. Mais seul Le Monde va l'utiliser. En effet, Libération est dans une situation financière plus critique et une grève mènerait de manière quasi inéluctable à la liquidation judiciaire. Un préavis de grève est déposé le 28 novembre 2006, mais le principe est rejeté en assemblée générale le 4 décembre (alors que c'est un instrument qui avait été utilisé lors du précédent plan de sauvegarde de l'emploi, qui pourtant concernait le départ d'un nombre inférieur de salariés, avec quatre jours de grève en novembre 2005). Cela montre la force de la pression qui pèse sur les salariés et leurs représentants ; ils ne veulent pas

(12) Article L. 1233-35 du Code du travail : lorsque le comité d'entreprise a recours à l'assistance d'un expert-comptable, la première réunion du comité d'entreprise est gelée. Une seconde réunion doit être organisée dans un délai de 20 à 22 jours.

(13) Comme le montrent Adam et Reynaud (1978, p. 300-301) dans leur exemple sur le secteur de la chaussure à Fougères (Bretagne), le fait que les syndicats soient ou non divisés est un élément qui influence le devenir d'une négociation. 
risquer de mettre en péril la survie du journal. Mais certains syndicats (dont la CGT) appellent tout de même à la grève : «Je préfère rien à un Libé complètement dénaturé » déclare un syndicaliste (au cours d'un entretien). Au contraire, au Monde, la grève est largement acceptée, car l'entreprise est dans une situation économique moins dangereuse. Les trois jours de grève (14 avril, 16 avril, 5 mai 2008) visent à faire accepter par la direction la transformation du plan de départs contraints en un plan de départs volontaires. La mobilisation des salariés prend alors des formes originales. Par exemple, les salariés défilent avec des masques blancs sur le visage, numérotés de 1 à 129 (pour le nombre de postes supprimés).

Cependant, les syndicats agissent aussi par le dialogue. La discussion autour d'un plan de départs volontaires au Monde en est emblématique (avrilmai 2008). Les discussions au sein du comité d'entreprise sont encadrées par le droit : en effet, les salariés licenciés sont censés être choisis selon des critères objectifs répondant à ce que l'on nomme l' « ordre des licenciements », pour que le plan soit validé par l'inspection du travail. Pour mettre en place un plan de départs volontaires, les discussions avec les syndicats se font en marge de la procédure légale d'information-consultation du comité d'entreprise. La partie intitulée «mesures de nature à limiter le nombre de licenciements » du Livre IV fait l'objet d'un accord parallèle avec les syndicats. " On s'est quand même vite rendu compte que le plan ne serait pas négocié par le comité d'entreprise mais directement avec les salariés » racontent les membres du comité d'entreprise. En dissociant le processus juridique et les négociations informelles, les partenaires sociaux ont pu trouver un accord. " Traditionnellement dans les journaux il y a toujours eu des niveaux d'extra-légal [négociations informelles en dehors du cadre juridique] qui étaient importants » raconte l'expert mandaté par le comité d'entreprise du Monde.

L'action légale du comité d'entreprise n'en est pas pour autant disqualifiée. Ce sont les mêmes interlocuteurs au comité d'entreprise et dans les syndicats. Il s'agit d'une utilisation stratégique des entités juridiques « comité d'entreprise » et «syndicats », et non d'une gestion différenciée du plan par des acteurs différents, qui est à l'origine de ces avancées.

\section{Un registre d'action classique ?}

Concrètement, ces négociations ont permis de réelles avancées sur le contenu des plans sociaux. À Libération, les salariés ont obtenu que le plan soit découpé en deux phases. Sur les 81 départs prévus, 18 sont mis de côté pour être soumis à une expertise nouvelle technologie (par le comité d'hygiène, de sécurité et des conditions de travail - CHSCT) $\mathrm{au}$ sein du projet Méthode. Au Monde, après

\section{Encadré 4}

\section{Indemnités de départ pour les rédacteurs du Monde}

Dans la convention collective, les journalistes touchent un mois de salaire par année d'ancienneté jusqu'à quinze ans d'ancienneté, et au-delà de quinze ans d'ancienneté, un comité arbitral décide de la somme à verser (en général, moins d'un mois par an). La direction du Monde propose des indemnités plus importantes que celles prévues par la convention collective.

\begin{tabular}{|l|c|c|c|c|}
\hline \multicolumn{1}{|c|}{ Ancienneté } & $\begin{array}{c}\mathbf{0 - 2 0} \\
\text { ans }\end{array}$ & $\begin{array}{c}\mathbf{2 1 - 2 5} \\
\text { ans }\end{array}$ & $\begin{array}{c}\mathbf{2 6 - 3 0} \\
\text { ans }\end{array}$ & + 30 ans \\
\hline $\begin{array}{l}\text { Mois de salaire } \\
\text { par année } \\
\text { d'ancienneté }\end{array}$ & 1,2 & 1 & 0,75 & 0,2 \\
\hline
\end{tabular}

Par exemple, pour un salarié avec quinze ans d'ancienneté (ancienneté moyenne au journal), la convention collective prévoit une indemnité de quinze mois de salaire. Si le salarié est candidat au départ volontaire, son indemnité sera de dix-huit mois de salaire.

Sources : entretiens, rapports d'experts.

négociations, le nombre de postes supprimés passe de 129 initialement prévus à 105 effectifs. Mais plus que ces gains en termes de postes, les négociations ont, dans les deux cas, permis le passage d'un plan de départs contraints à un plan de départs volontaires. Un 《 guichet départ volontaire » est ouvert en janvier 2007 à Libération et en mai 2008 au Monde pour toutes les catégories de personnels. Les indemnités de départ sont avantageuses (et donc supérieures à celles garanties par les conventions collectives qui sont appliquées en cas de départs contraints) afin d'inciter les gens à partir ( $c f$. encadré $4)$.

Certains bénéficient alors d' « effets d'aubaine », c'est-à-dire que certains salariés qui ne seraient pas partis avec un plan de départs contraints (notamment les plus âgés qui sont très protégés par la loi) vont s'inscrire au « guichet départ » et bénéficier d'une très forte indemnité, à l'exemple de cette journaliste (21 ans d'ancienneté) : «Je suis partie avec le dernier plan [départ le 31 décembre 2008]. Cela m'a permis de prendre ma retraite un peu en avance, en octobre 2009. C'est une des raisons pour lesquelles je me suis décidée. J'avais des conditions économiques et sociales très favorables entre les congés payés, les indemnités chômage pour les plus de 57 ans et les indemnités de licenciement [...]». (Entretien avec une ancienne journaliste du Monde).

Ainsi, la négociation a un effet réel sur la détermination des personnes licenciées et sur les indemnités de départ. Cependant, la portée de ces avancées est à nuancer, puisque les salariés ne remettent pas fondamentalement en cause les choix de la direction. Les modalités d'application du plan ont été infléchies, mais des départs ont effectivement eu lieu 
Encadré 5

Bilan des départs

\begin{tabular}{|c|c|c|c|c|c|c|c|c|c|c|c|}
\hline & \multicolumn{5}{|c|}{ Libération } & \multicolumn{6}{|c|}{ Le Monde } \\
\hline & 2006 & 2007 & & Variation & & 2007 & & 2009 & & Variatior & \\
\hline & Effectif । \% & Effectif & $\%$ & Effectif & $1 \%$ & Effecti & fi $\%$ & Effectif| & $\%$ & Effectif & $1 \%$ \\
\hline Journaliste & \begin{tabular}{l|l}
214 & 75,4
\end{tabular} & 173 & 177,2 & 41 & 19,2 & 341 & 58,4 & 272 & 158,9 & 69 & 20,2 \\
\hline Cadre & $41 \quad$ I 14,4 & 32 & 14,3 & 9 & 122,0 & 150 & 125,7 & 122 & 26,4 & 28 & $\mid 18,7$ \\
\hline $\begin{array}{l}\text { Ouvrier / } \\
\text { Employé }\end{array}$ & 29 & 19 & 8,5 & 10 & 134,5 & & 15,9 & & 13,4 & & $\begin{array}{l}1 \\
133,3\end{array}$ \\
\hline Hommes & $158 \quad$ । 55,6 & 129 & 57,6 & 29 & $\mathbf{1 1 8 , 4}$ & 323 & 155,3 & 250 & 54,1 & 73 & I22,6 \\
\hline Femmes & $128 \quad$ I 45,1 & 95 & 42,4 & 33 & 25,8 & 261 & 44,7 & 212 & 45,9 & 49 & 18,8 \\
\hline Age moyen & 44,13 & 43,66 & & 0 & & n. a. & & 47,95 & & n.a. & \\
\hline Ancienne té & 13,87 & 12,91 & & 1 & & n. a. & & 15,36 & & n.a. & \\
\hline Rémunération & 3704,5 & 3784 & & $-79,5$ & I & n. a. & & 5302 & & n.a. & \\
\hline Total & 284 & 224 & & 60 & & 584 & & 462 & & 122,0 & \\
\hline
\end{tabular}

Sources : bilans sociaux, au 31 décembre de l'année.

Note : à Libération, le plan social est négocié en 2006 et les départs ont lieu entre mars et juin 2007. Au Monde, le plan social est négocié début 2008 , et les départs s'étalent entre avril 2008 et mars 2009

À Libération, le plus grand nombre de départs (en effectif) a lieu chez les journalistes, mais, proportionnellement, c'est la catégorie employé/ouvrier qui est la plus touchée par les départs (une baisse d'effectifs de $35 \%$ dans cette catégorie, contre environ $20 \%$ de départs chez les journalistes et les cadres).

(cf. encadré 5). Les plans signés sont des compromis issu de la négociation collective, et bénéficient du soutien de tous : «le meilleur plan, c'est celui qui a été signé par tous les partenaires » explique un membre de la direction.

Dans ces deux entreprises, la nécessité d'une restructuration n'est pas remise en cause. Le travail de l'expert économique, mandaté par le comité d'entreprise, est très important, car il confirme la validité des chiffres avancés par la direction. Les revendications se cristallisent autour des modalités de départ et notamment autour du volontariat. Si l'on reprend la typologie des registres d'action des salariés en restructuration, on peut relier ces deux cas de restructuration à l'idéal type « approfondissement des politiques de l'emploi ». Cependant, les salariés s'inscrivent également dans la « critique de la direction ». Sans remettre en cause la nécessité d'une restructuration, le comité d'entreprise conteste la manière dont les réductions de coûts sont appréhendées. La direction est dans une logique de nombre de postes supprimés, alors que les représentants des salariés mettent en avant le nombre de postes nécessaires pour faire un journal. Tout en acceptant la recherche d'une économie sur la masse salariale, ils refusent une logique de suppression d'emplois qui ne prenne pas en compte les besoins rédactionnels.

Au-delà de ces négociations classiques sur les modalités d'application du plan, les salariés vont se placer sur des terrains non économiques qui dépassent leurs prérogatives légales afin d'influencer le devenir du produit, c'est-à-dire la forme et le contenu du journal.

\section{Restructurations, innovations, transactions}

Les salariés et les dirigeants de Libération et du Monde sont confrontés à une crise de rentabilité qui traduit les difficultés du modèle économique de leur entreprise. À cette occasion, une réflexion est engagée quant au produit, à l'organisation et aux valeurs de l'entreprise, en réaffirmant le statut spécifique du journaliste et son interdépendance avec les autres salariés. Le comité d'entreprise devient un lieu de débat qui dépasse les prérogatives légales d'information-consultation portant sur l'activité économique de l'entreprise : «En CE on parle de choses dont on ne devrait pas nécessairement parler en CE » raconte un élu de Libération. Dans ces deux entreprises, les débats ont porté sur deux questions fondamentales : la qualité rédactionnelle du titre et la gestion de son actionnariat. Une troisième question se pose dans le cas du Monde : celle des frontières du Groupe La Vie-Le Monde.

\section{Une nouvelle formule rédactionnelle}

Les journalistes partagent des catégories de pensée permettant de faire la distinction entre le « bon » journalisme et le « mauvais » (PILmis, 2007). Ces catégories fondent les valeurs mobilisées par les rédacteurs : clarté, indépendance, bonne 
foi, vérification, originalité. Des transformations se sont opérées dans les façons de travailler sous les pressions du marché et du lectorat. Par exemple, au Monde, Eugénie SAÏTTA (2005) souligne le passage d'une « rhétorique de l'expertise critique » (journalisme documentaire, vérification et éclaircissement d'une information) qui prédominait dans les années 1980 (14), à une « rhétorique de la révélation » (du scoop) dans les années 2000, dans laquelle la quantité d'information produite importe davantage que sa qualité et sa justesse, comme en témoigne cette journaliste : «Moi quand je suis rentrée, on ne publiait pas une brève sans avoir passé deux coups de fil pour la vérifier. Aujourd'hui on recopie les dépêches AFP [Agence France-Presse] ». (Entretien avec une ancienne journaliste du Monde).

Les impératifs économiques obligent les quotidiens à se placer dans une logique de production qui tend à uniformiser le traitement de l'information. Malgré ces transformations, les valeurs mobilisées par les journalistes restent les mêmes. L'originalité et la qualité du projet éditorial demeurent le défi à relever pour assurer l'avenir des journaux (PIGEAT, PARACUEllons, 2006). Or la question se pose de la capacité à faire un journal de qualité après autant de départs. Malgré les réductions d'effectifs, les journalistes veulent conserver leur modèle d'excellence professionnelle.

"On était 330 salariés il y a un an, si le plan Rothschild passe, on sera 180. [...] Un journaliste peut écrire 4500 mots au lieu de 1500 pour un article, c'est facile, il suffi t de délayer, mais ça perd en qualité ». (Entretien avec un journaliste de Libération).

Les deux plans sont accompagnés de la mise en place d'une nouvelle formule éditoriale censée répondre aux attentes des lecteurs. Sur cette base, les instances représentatives du personnel alimentent le débat autour du projet rédactionnel en poussant la direction à l'expliciter (afin de justifier les licenciements) et influencent donc ce que sera la « nouvelle formule ». L'ensemble des salariés sont investis dans cette négociation. Bien que le rédactionnel soit proprement journalistique, les autres salariés se sentent concernés par la « qualité rédactionnelle de leur titre ».

«On est fier d'appartenir à cette maison [...]. Quand on dit qu'on est salarié au journal Le Monde, on sent les yeux qui brillent, on a la sensation qu'on appartient à quelque chose d'exceptionnel ». (Entretien avec une employée du Monde).

On peut étudier la discussion autour de la nouvelle formule à Libération. Même si l'identité collective

(14) Par rapport à une étude datant de 1985 : PADIOLEAU, 1985. du journal Libération a évolué (15), les salariés se sentent impliqués dans la sauvegarde de celle-ci. De manière générale, les journalistes imposent l'idée qu'ils ne font pas « un métier comme les autres » (LEMIEUX, 1994).

"C'est une entreprise très particulière, les gens sont très attachés au journal, à ce qu'il dit, à ce qu'il fait dire. Il y avait des assemblées générales toutes les semaines, des débats ». (Entretien avec un délégué syndical de Libération).

Avec la suppression de 81 postes et l'introduction d'un nouveau logiciel d'édition, l'offre se standardise (avec le projet Méthode qui introduit un nouveau système informatique d'édition). L'introduction d'une nouvelle formule bouscule les routines journalistiques. Le comité d'entreprise devient le lieu de débats sur la définition du produit (avenir du journal). En outre, l'expert est censé évaluer la situation financière du journal. Cependant, il prend en compte dans son rapport des éléments concernant la qualité - et non pas seulement la faisabilité - du journal. Le recours à l'expert permet ainsi que certains postes soient conservés et certains départs différés. Dans le cadre du projet Méthode, les élus du personnel ont saisi un expert « nouvelle technologie » (dans le cadre du CHSCT), pour les 18 postes supprimés à cause du logiciel. Comme dans le cas de la saisie d'un expert économique par le comité d'entreprise, la demande d'un rapport CHSCT gèle l'avancée du plan social tant que le rapport n'est pas rendu (avril 2007).

"Quand vous avez un problème et que vous voulez gagner du temps, exigez un bilan CHSCT, il y a toujours une bonne raison, et ça vous fait gagner un mois et demi ! » (Entretien avec un délégué syndical de Libération.)

Cette astuce a permis de réajuster le plan de sauvegarde de l'emploi discuté dans un premier temps, puisque les 18 départs concernés ont été retardés (de mars à juin 2007).

\section{La neutralité actionnariale}

La « vénalité de la presse » est régulièrement dénoncée (Martin, 2006). Pourtant les journalistes revendiquent leur indépendance (financière et éditoriale). Or les actionnaires commencent à demander des comptes. La question se pose d'une éventuelle recapitalisation : les arrivées d'actionnaires qui ne sont pas « neutres » (Rothschild à Libération et Lagardère au Monde) posent problème, d'autant plus que les sociétés de salariés-actionnaires perdent de leur pouvoir (avec la modification des statuts de la SCPL en janvier 2007 et la dilution du capital de
(15) Avec, selon la thèse de Gérard Rimbert (2005), la transformation d'un groupe d'écrivains maoïstes en journalistes professionnels payés par le grand capital, soit le passage « de Sartre à Rothschild ». 
la SRM). Au Monde, l'identité d'un éventuel futur actionnaire est conditionnée par les valeurs morales que portent les salariés du journal. Il faut que l'actionnaire soit lié au monde de la presse.

«Si on veut être journaliste, il faut être entrepreneur pour pouvoir garder son indépendance. On aurait pu considérer que ce n'est pas notre métier de faire de la gestion et laisser faire des grosses boîtes, mais alors qu'est ce qu'on fait de notre indépendance? ». (Entretien avec un membre de la direction du Monde.)

La direction et ses salariés s'entendent sur la nécessité de conserver une presse indépendante, comme gage de qualité. Les instances de représentation du personnel sont très impliquées dans ce choix d'éventuels nouveaux actionnaires : la SRM en tant qu'actionnaire, mais aussi les autres. Les journalistes menacent d'utiliser la clause de conscience en cas de perte de l'indépendance du quotidien. Ils utilisent cette opportunité juridique pour peser sur les négociations et ainsi lutter contre le managérialisme, défini par Fabien Blanchot et Jean-Gustave Padioleau comme "la compétition généralisée et impérative qui imprègne les firmes » (p. 68). Les salariés opposent à « l'identité sociale réelle » de l'entreprise (sa position économique, c'est-à-dire ses finances) son «identité sociale virtuelle » (ses valeurs fondées sur ses routines, son histoire), en mettant en avant les croyances et les traditions telles que l'héritage des fondateurs (ibid. p. 70).

Dans son chapitre consacré à Libération et Le Monde (p. 192-209), Julien Duval (2004) met au jour la progression d'une «idéologie commerciale » en parallèle de l'arrivée d'investisseurs privés et de la transformation de ces entreprises en « grande entreprise privée capitaliste ». La production journalistique est selon lui le relais direct de l' " économie libérale ». Au contraire, notre travail ne montre pas la disparition d'une idéologie «morale » au profit (et au service) d'une logique commerciale, mais montre comment les acteurs font coexister ces deux logiques. Il y a une concurrence entre deux formes de rationalité. Les salariés poussent vers une gestion rationnelle «matérielle » qui permet la coexistence de valeurs diverses dans la pratique (économiques, éditoriales, éthiques...) plutôt que « formelle » qui mettrait l'accent uniquement sur les impératifs économiques (STEINER, 1998).

\section{Redéfinir les frontières}

Dans le cas du Monde, l'un des enjeux de la restructuration est la redéfinition du périmètre du Groupe La Vie-Le Monde, par la cession de filiales déficitaires. La gestion des filiales par Le Monde est emblématique de la singularité des entreprises de presse, prêtes à tout pour sauvegarder le journal.

Les cessions correspondent à un recentrage de ce Groupe de presse autour des activités du quotidien. Les filiales sont utilisées lors de l'achat et de la vente, comme des investissements financiers permettant de renflouer le quotidien. Elles ne sont jamais envisagées comme des investissements rédactionnels. Ce sont des "pépites financières » qui servent à équilibrer les comptes du quotidien. Le Groupe n'a jamais eu de politique d'intégration des filiales, mais seulement un comportement de « prédation ». Au cours du plan de sauvegarde de l'emploi, les reclassements au sein du Groupe ne sont pas envisagés.

La Société éditrice du Monde (SEM) étant la structure porteuse des filiales, la cession de filiales la concerne en premier lieu. Cependant, ces cessions modifient les frontières du Groupe ce qui explique que la vente des filiales soit débattue en conseil de surveillance (au niveau du Groupe). Une filiale ne peut pas être vendue sans l'aval du conseil de surveillance où les sociétés de personnels du Monde disposent de 6 voix sur 20. Dans la pratique, les sociétés de personnels du Monde n'entravent pas la validation des cessions. Les filiales en vente souhaitant être vendues, les personnels du quotidien respectent la décision des filiales en validant les cessions.

Cependant, les sociétés de personnels sont actionnaires c'est-à-dire qu'elles doivent veiller à la viabilité financière de l'entreprise. Que se serait-il donc passé si une filiale fortement déficitaire avait souhaité rester dans le Groupe ? Les sociétés de personnels auraient-elles voté contre la cession ? La politique affichée consiste à voter comme les salariés concernés, mais elle pourrait rentrer en contradiction avec la défense de la valeur pour l'actionnaire dans le cas où les salariés des filiales concernées auraient voté pour un maintien de leur entreprise dans le Groupe. Une fois les ventes validées en conseil de surveillance, les plans de sauvegarde de l'emploi présentés dans les filiales sont directement négociés par les salariés des filiales.

Les frontières du Groupe tendent ainsi à se refermer sur le quotidien par la cession des filiales, en limitant ainsi la portée d'un comportement prédateur au service de celui-ci.

\section{La redécouverte du registre transactionnel}

Les discussions entre les salariés et la direction ne s'arrêtent donc pas aux modalités d'application du plan. Au-delà des modalités concrètes d'application $\mathrm{du}$ plan (propositions économiques alternatives), c'est l'avenir de l'entreprise (périmètre, actionnariat) et de son produit (formule) qui est discuté par les représentants des salariés. Les discussions ont de réelles portées concrètes quant à la définition de ces éléments : les nouvelles formules sont retravaillées, notamment à Libération avec l'intervention du CHSCT ; les actionnaires qui entrent au capital après la recapitalisation s'engagent à ne pas intervenir dans l'éditorial ; le périmètre de l'entreprise est redéfini. 
Cela tient à ce que les relations de travail dans ces entreprises engagent plus qu'une relation d'emploi, elles sont dominées par une éthique professionnelle. Les valeurs mobilisées au travail pèsent ainsi sur le cours des restructurations puisqu'elles sont à la base de l'argumentaire déployé par les salariés. On peut relier ces deux cas de restructuration à l'idéal-type du « registre transactionnel », construit à partir d'une investigation sur les restructurations au sein de Canal Plus (DIDRY, 2007). Ce registre lie négociation sur les volets économiques et discussion sur l'avenir de l'entreprise et de son activité. Comme dans le cas de Canal Plus, les instances de représentation du personnel du Monde et de Libération passent du débat au dialogue. D'une négociation (recherche d'un accord pour résoudre un conflit), les salariés arrivent à un processus de délibération (discussion collective en vue d'une décision à prendre en commun : URFALINO, 2005). Ce registre semble être caractéristique des entreprises dans lesquelles l'implication des salariés dans la réalisation du produit est très forte, ce qui pousse les directions d'entreprise à agir sur un mode plus coopératif (on retrouve ici le parallélisme esquissé en première partie entre les coopératives et les sociétés de personnels actionnaires).

"Participer dans le sens positif du terme, parce que les journalistes ont plein de savoirs, c'est dommage de s'en priver. » (Entretien avec le président du forum des SDJ (16)).

En se rapportant à trois « registres d'action des salariés », ces cas s'inscrivent dans une « combinaison entre différentes logiques d'action [les différents registres] qui coexistent »(BASZANGER, DODIER, 1997). Cette typologie est opératoire pour penser la diversité des dynamiques salariales en lien avec les singularités des «mondes de production ». Plus que le monde de la presse, ce registre se retrouve dans les mondes dans lesquels les salariés sont fortement engagés dans la gestion de leur entreprise, de manière à la fois légale (actionnariat salarié) et morale (éthique professionnelle au cœur de leur identité). Ces deux entreprises répondent bien à ces critères, avec des instances de représentation du personnel spécifiques, le brouillage des frontières entre les différents représentants, le poids de la rationalité axiologique par rapport à une rationalité économique, et la grande part de l'« extra-légal » dans les discussions.
L'étude de la négociation et de la mise en place de plans de sauvegarde de l'emploi à Libération et au Monde permet de valider l'hypothèse d'une influence des institutions du travail sur les activités économiques et précisément dans ces cas à travers la mobilisation des règles par les salariés. En effet, le devenir des entreprises est influencé par le fait que les acteurs utilisent de manière détournée les cadres juridiques et les règles de droit (consultations légales, clause de conscience, actionnariat salarié...), afin de satisfaire leur «mythologie professionnelle » (Le BoHec, 2000). Parmi l'éventail des possibles, les salariés ont choisi une manière spécifique de se mobiliser qui donne des résultats uniques à ces restructurations. Ainsi, la mise en place du plan - que ce soit sur le volet économique et social (classique), ou sur le volet de l'avenir du produit et de l'entreprise (singulier) - est influencée par ces choix des salariés. Ces choix sont liés à leur éthique professionnelle, à la structure du capital des entreprises et à leur mode particulier de représentation. Cette étude cerne avec plus de précision l'un des registres d'action des instances de représentation du personnel, le « registre transactionnel ». Identifié dans trois entreprises de médias (Canal Plus dans DiDRY, 2007 ; Le Monde et Libération ici), il semble être caractéristique des entreprises dans lesquelles les salariés disposent de moyens d'action puissants (salariés actionnaires) et présentent un fort investissement dans la production du contenu. Ils s'impliquent dans la gestion de leur entreprise de manière plus citoyenne (défense d'une démocratie) que professionnelle (défense de son emploi) et coopèrent à la détermination de leur avenir. Ainsi la sociologie des entreprises de presse doit-elle inventer des outils ajustés aux singularités de ce monde de production et de son salariat. 


\section{Bibliographie}

Adam G., Reynaud J.-D. (1978), Confl it du travail et changement social, Paris, PUF.

Amado-Borthayre L. (2009), « Enjeux de gouvernance dans une coopérative multinationale », Pôle Sud, no 31, pp. 87-102.

Aubert J.-P., Beaujolin-Bellet R. (2004), « Les acteurs de l'entreprise face aux restructurations : une délicate mutation », Travail et Emploi, no 100, pp. 99-112.

BAstin G. (2001), « La presse au miroir du capitalisme moderne. Un projet d'enquête de Max Weber sur les journaux et le journalisme », Réseaux, no 2001/5, pp. 172-208.

Baszanger J., Dodier N. (1997), « Totalisation et altérité en sciences sociales », Revue française de sociologie, no 38-1, pp. 37-66.

Bernard DE RAYMond A. (2004), « La règle de droit comme maxime empirique de l'activité économique. À propos de Rudolf Stammler et le matérialisme historique de Max Weber (note critique) », Terrains et Travaux, no 6 , pp. 71-80.

Blanchot F., Padioleau J.-G. (2003), « Une économie politique du travail journalistique », Hermès, 35, pp. 63-71.

BocquetA.-M., Gerardin H.,Poirot J.(2010), «Économie sociale et solidaire et développement durable : quelle spécificité pour les coopératives et les mutuelles ? », Géographie, économie et société, 12, pp. 329-352.

Boyer T. (2005), « Déconstruction du projet de licenciement », Revue de l'IRES, vol. 47, $\mathrm{n}^{\circ}$ 1, pp. 175-193.

Cabrolie S. (2009), « La double vente du journal quotidien » in VATIN F. (éd.), Évaluer et valoriser, une sociologie économique de la mesure, Toulouse, Presses universitaires du Mirail, pp. 177-195.

Champagne P. (2000), « Le médiateur entre deux Monde », Actes de la recherche en sciences sociales, vol. 131-132, pp. 8-29.

Coenen-Huther J. (2007), « Classifications, typologies et rapport aux valeurs », Revue européenne des sciences sociales, numéro XLV-138, pp. 27-40.

Cohen P., Pean P. (2003), La face cachée du Monde, Paris, Fayard.

Da Lageo O. (2003), Obtenir la carte de presse et la conserver, Paris, Victoires Éditions.

De Saint-Laurent-Kogan A.-F. (2000), « Qui fait quoi », Actes de la recherche en sciences sociales, vol. 134, pp. 56-61.

Delporte C. (1999), Les journalistes en France 18801950 : naissance et constitution d'une profession, Paris, Le Seuil.

Duval J. (2004), Critique de la raison journalistique. Les transformations de la presse économique en France, Paris, Le Seuil.
DIDRY C. (1998), « Les comités d'entreprise face aux licenciements collectifs : trois registres d' argumentation », Revue française de sociologie, $\mathrm{n}^{\circ} 39-3$, pp. 495-534.

DIDRY C. (2007), « De la restructuration dans l'entreprise à la restructuration des relations professionnelles : le cas de Canal Plus », L'Homme et la Société, no 63-64, pp. 95-124.

Guisnel J. (2003), Libération : la Biographie, Paris, La Découverte.

Eveno P. (1997), « Comment Le Monde est-il entré en entreprise ? », Matériaux pour l'histoire de notre temps, vol. 46, no 46, pp. 27-31.

Eveno P. (2004), Histoire du journal Le Monde (19442004), Paris, Albin Michel.

Le Bohec J. (2000), Les mythes professionnels des journalistes, Paris, Broché.

Lemieux C. (1992), « Les journalistes, une morale d'exception ?», Politix, vol. 5, no 19, pp. 7-30.

Lemieux C. (2000), Mauvaise presse, Paris, Édition Métaillé.

Martin M. (2006), « Retour sur l'abominable vénalité de la presse », Le Temps des Médias, 2006/1, nº 6, pp. 22-33.

Neveu E. (2004), Sociologie du journalisme, Paris, La Découverte.

Rimbert P. (2005), Libération : de Sartre à Rothschild, Paris, Raisons d'agir.

Padioleau J.-G. (1985), Le Monde et le Washington Post, précepteurs et mousquetaires, Paris, PUF.

Pigeat H., Paracuellos J.-C. (2006), « Les marchés de la presse quotidienne en Europe », Le Temps des Médias, 2006/1, n 6, pp. 72-86.

Pilmis O. (2007), « Faire valoir ses compétences : les pigistes et le placement du sujet », Formation Emploi, 99 , pp. 75-87.

SaItTA E. (2005), «Le Monde, vingt ans après », Réseaux, 2005/3, no 131, pp. 189-225.

SAlais R., Storper M. (1993), Les mondes de production : enquêtes sur l'identité économique de la France, Paris, Éditions EHESS.

Steiner P. (1998), Sociologie de la connaissance économique. Essai sur les rationalisations de la connaissance économique, 1750-1850, Paris, PUF.

URfalino P. (2005), « La délibération n'est pas une conversation », Négociations, (2), pp. 99-114.

Weber M. (1910), Rapport préliminaire pour une proposition d'enquête sur la sociologie de la presse, rédigé par Weber au début de 1910 et distribué aux personnes pressenties pour le projet, traduit en annexe de Bastin G. (2001). 